\title{
Designs and Methodologies Used in Landmark Clinical Trials of Glaucoma: Implications for Future Big Data Mining and Actionable Disease Treatment
}

\author{
Saif Aldeen AlRyalat ${ }^{1 *}$, Monica K. Ertel ${ }^{2}$, Leonard K. Seibold ${ }^{2}$ and Malik Y. Kahook ${ }^{2}$ \\ ${ }^{1}$ Department of Ophthalmology, The University of Jordan, Amman, Jordan, ${ }^{2}$ Department of Ophthalmology, Sue \\ Anschutz-Rodgers Eye Center, University of Colorado School of Medicine, Aurora, CO, United States
}

Keywords: glaucoma, open angle, clinical trials, diagnosis, optic nerve

\section{INTRODUCTION}

A widely agreed upon definition of glaucoma with clear diagnostic criteria to classify disease presence and status remains elusive in both clinical and research settings. For decades, the diagnosis

OPEN ACCESS

Edited by:

Luciano Quaranta,

University of Pavia, Italy

Reviewed by:

Angelina Tzacheva,

University of North Carolina at

Charlotte, United States

*Correspondence:

Saif Aldeen AlRyalat

s.alryalat@ju.edu.jo;

saifryalat@yahoo.com

Specialty section:

This article was submitted to

Ophthalmology,

a section of the journal

Frontiers in Medicine

Received: 19 November 2021 Accepted: 04 January 2022 Published: 26 January 2022

Citation:

AlRyalat SA, Ertel MK, Seibold LK and Kahook MY (2022) Designs and

Methodologies Used in Landmark Clinical Trials of Glaucoma: Implications for Future Big Data Mining and Actionable Disease Treatment. Front. Med. 9:818568. doi: 10.3389/fmed.2022.818568 of glaucoma was primarily based on documenting visual field changes through static or kinetic perimetry and correlating these findings with structural changes at the optic nerve head $(1,2)$. Recently, the 10th World Glaucoma Association (WGA) Consensus Meeting supported the use of optic nerve structural endpoints alone to provide sufficient information for a diagnosis of glaucoma, even in the absence of visual field changes, a state termed pre-perimetric glaucoma (3). The National Institute for Health and Care Excellence (NICE), the national body tasked with issuing clinical guidelines for England and Wales, does not provide strict criteria to diagnose glaucoma (4). There is scarce guidance on criteria to definitively diagnose glaucoma which leads to difficulties in research efforts focused on phenotyping ocular imaging/diagnostic data as a first step toward identifying and predicting disease to enhance clinical care.

\section{LANDMARK TRIALS IN GLAUCOMA}

The field of glaucoma has the second largest number of published randomized controlled trials in all of ophthalmology, the majority of which evaluate glaucoma treatments $(5,6)$. Landmark randomized controlled trials have shaped the practice of glaucoma care and are commonly used to teach medical learners the basics of treating patients with suspected or diagnosed glaucomatous optic neuropathy (7-10). While it is clear that these trials have provided a wealth of information for practical patient care, the added benefit of these data sets is the ability to mine information that can guide the planning of future studies. This can take the form of feeding large labeled data sets into novel machine learning algorithms which potentially can produce new findings to inform patient care $(11,12)$. The large data sets from previous landmark trials are often published in top tier journals and receive a great deal of attention. However, the building blocks of these studies; including methodologies, patient selection criteria and diagnostic operating procedures, are usually published in a separate document and receive less rigorous attention $(1,2,13)$. The aim of this brief report is to clarify the diagnostic criteria used by landmark glaucoma trials with a focus on design and methodology.

We reviewed diagnostic criteria used by landmark glaucoma clinical trials commonly cited in ophthalmic textbooks and review articles $(7-10)$. We also supplemented the mentioned trials by 
TABLE 1 | Landmark glaucoma clinical trials and each of the functional, structural, and intraocular pressure criteria used in glaucoma diagnosis or enrollment.

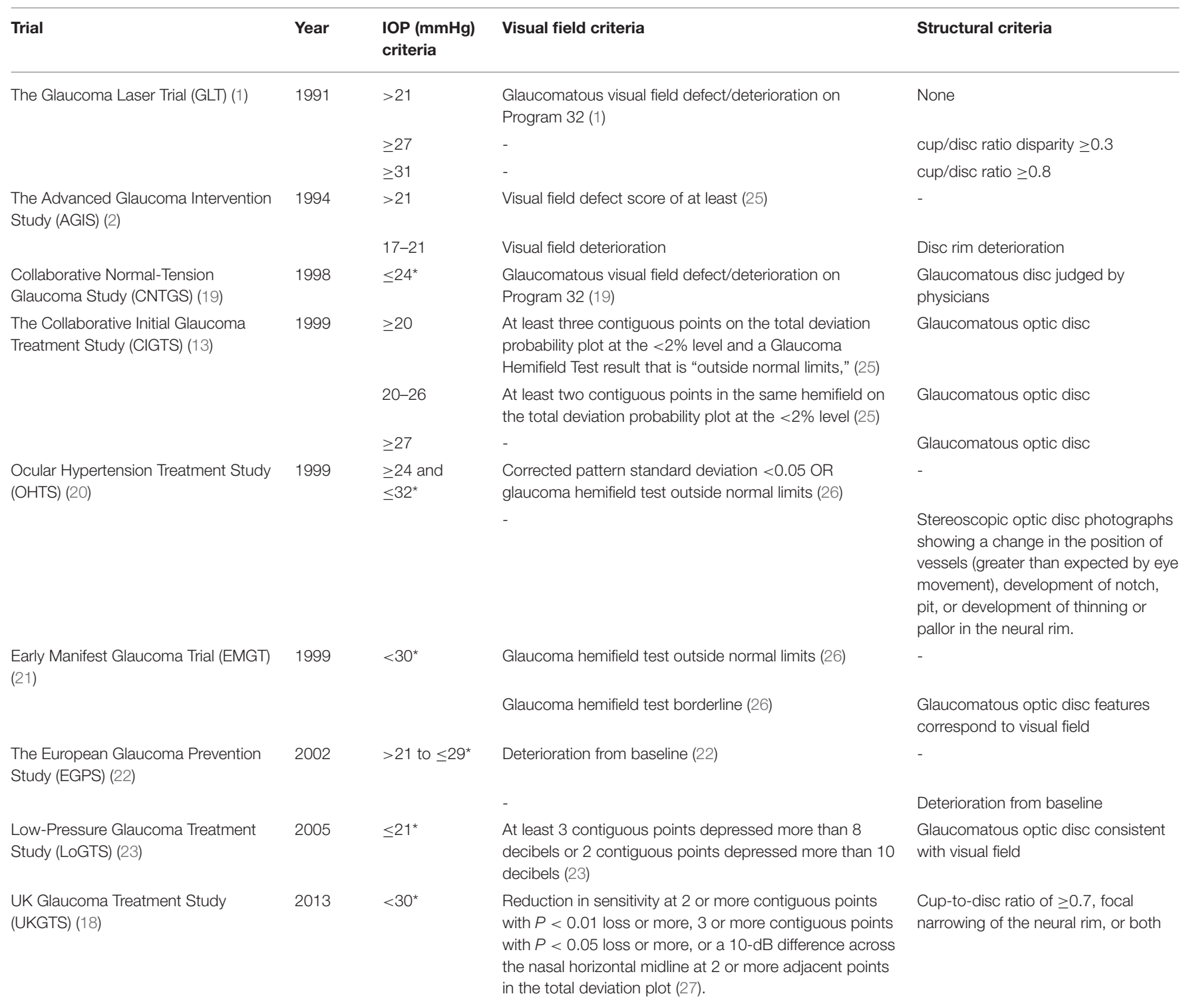

"These intraocular pressure thresholds were used for enrollment, rather than for establishing the diagnosis.

an advanced PubMed search for design and methodology articles published for glaucoma clinical trials where we used the following search strategy:

((Design[Title]) OR (Method[Title])) AND (Glaucoma [Title])

We included trials that were concerned mainly with open angle glaucoma, as this represents the majority of the effort in this space. Studies that had design protocols published in a separate "design and methodology" article were of particular interest.

Fourteen trials were identified in our assessment, however, five of them were surgical trials focused on outcome comparisons and used criteria previously utilized in larger randomized clinical trials (14-18). As a result, we focused on nine clinical trials $(1,2,13,19-24)$. Glaucoma diagnosis was based on one or more of the following criteria: functional criteria in terms of visual field performance, structural criteria in terms of optic disc features, and/or intraocular pressure (IOP). Table 1 details the specific glaucoma diagnostic criteria adopted by each of the landmark clinical trials. Visual field criterion was a pre-requisite diagnostic criterion for most clinical trials, although they varied in their definition for what qualified as a glaucomatous visual field $(1,13,19,22,23,25,26)$. The Glaucoma Laser Trial (GLT) and the Collaborative Initial Glaucoma Treatment Study (CIGTS) did not require visual fields for patients with an IOP of 27 or higher, where only structural evidence of glaucomatous optic disc damage was required $(1,13)$. More recent trials, including the European Glaucoma Prevention Study (EGPS) (22), LowPressure Glaucoma Treatment Study (LoGTS) (23), and UK 


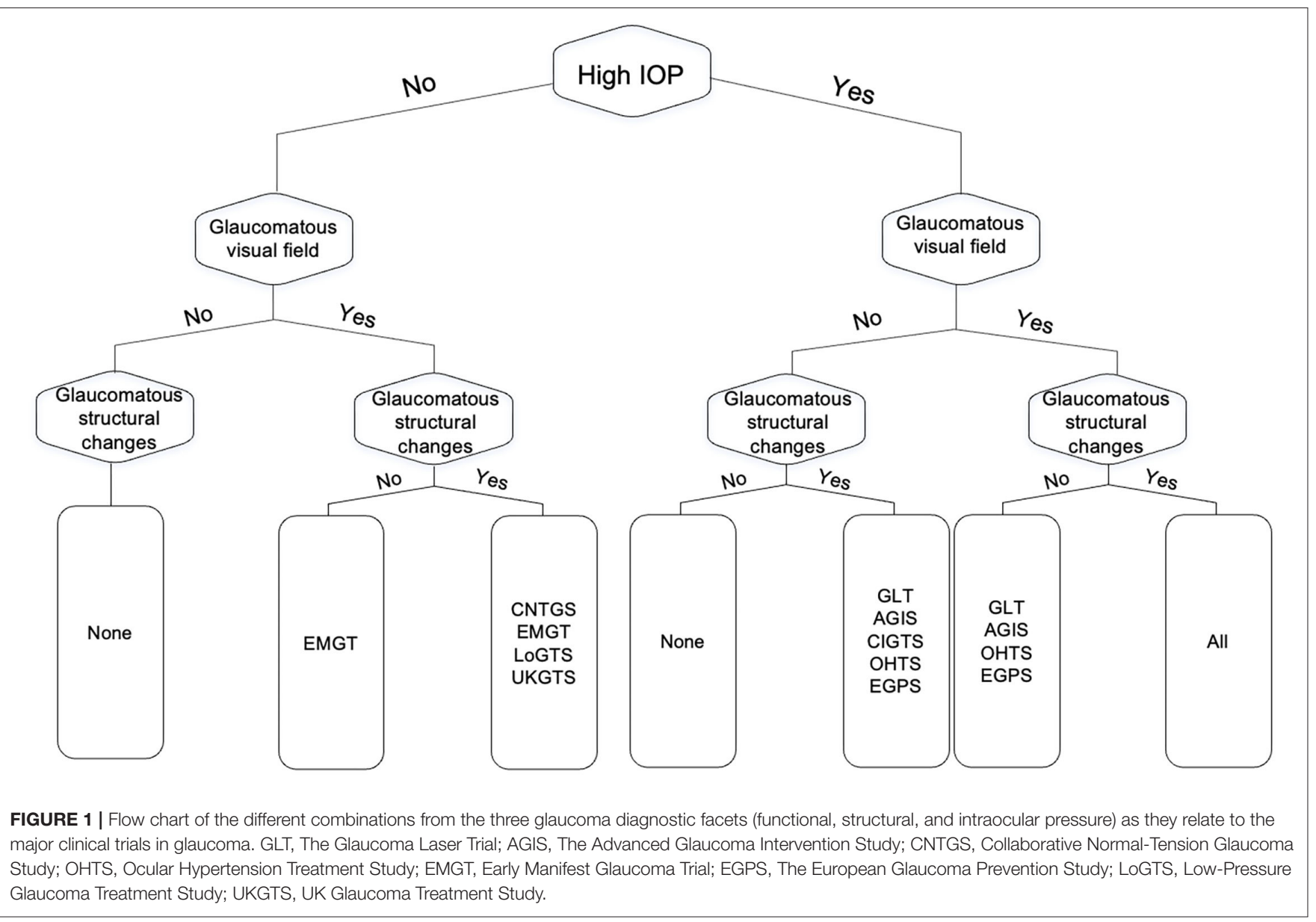

Glaucoma Treatment Study (UKGTS) (18), required structural glaucomatous features and/or visual field glaucomatous features. Figure 1 shows how the different combinations of functional, structural and IOP criteria were utilized across these studies.

\section{DISCUSSION}

Detailing the presence and/or progression of glaucomatous optic neuropathy is based on the functional and structural characteristics of the optic nerve, relying on the combination of both subjective and objective data obtained from clinical examination and output from various diagnostic modalities. While large randomized clinical trials have provided insights into the treatment of glaucoma, the criteria used for enrollment and interventions in each trial are disparate, making application of findings in the clinical setting difficult at best. With the emergence of artificial intelligence and machine learning techniques that seek to decipher new learnings from these large datasets, a deep and nuanced understanding of the designs and methodologies used is key to unlocking even more data to enhance patient care. We have provided an overview of diagnostic criteria used in landmark randomized controlled trials of open angle glaucoma. These criteria differ in the diagnostic weight placed on subjective visual field studies, objective structural changes of the optic nerve, as well as the use of IOP metrics. Outlining these criteria in a single resource may act as a starting point for discussions on proper methods of mining past data sets while also reaching some consensus for implementing a commonly agreed upon set of diagnostic criteria in future studies to facilitate broader analyses. The ultimate goal is to make findings from large randomized clinical trials more actionable in a real-world clinical setting by leveraging big data sets toward predictive output and guidance for when to observe and when to intervene with escalating care.

\section{AUTHOR CONTRIBUTIONS}

SA, ME, LS, and MK contributed in research conception, literature review, and manuscript writing. All authors contributed to the article and approved the submitted version. 


\section{REFERENCES}

1. The Glaucoma Laser Trial (GLT): 3. Design and methods. Glaucoma laser trial research group. Control Clin Trials. (1991) 12:504-24. doi: 10.1016/0197-2456(91)90010-J

2. Ederer F, Gaasterland DE, Sullivan EK. The Advanced Glaucoma Intervention Study (AGIS): 1. Study design and methods and baseline characteristics of study patients. Control Clin Trials. (1994) 15:299-325. doi: 10.1016/0197-2456(94)90046-9

3. Weinreb RN, Garway-Heath DF, Leung C, Medeiros FA, Liebmann J. Diagnosis of Primary Open Angle Glaucoma: WGA consensus series - 10. Kugler Publications (2017).

4. Overview | Glaucoma: diagnosis management | Guidance | NICE. Available online at: https://www.nice.org.uk/guidance/ng81 (accessed April 2, 2021).

5. AlRyalat SA, Abukahel A, Elubous KA. Randomized controlled trials in ophthalmology: a bibliometric study. F1000Res. (2019) 8:1718. doi: 10.12688/f1000research.20673.1

6. Storgaard L, Tran TL, Freiberg JC, Hauser AS, Kolko M. Glaucoma clinical research: trends in treatment strategies and drug development. Front Med. (2021) 8:733080. doi: 10.3389/fmed.2021.733080

7. Penman A, Crowder K, Watkins WM. 50 Studies Every Ophthalmologist Should Know. Oxford University Press (2020). doi: 10.1093/med/9780190050726.001.0001

8. Wishart PK. Interpretation of the glaucoma "landmark studies." $\mathrm{Br} J$ Ophthalmol. (2009) 93:561-2. doi: 10.1136/bjo.2008.145532

9. Henein C, Mathew RG. Use of infographics to communicate landmark glaucoma trials. Eye. (2021) 35:2073-4. doi: 10.1038/s41433-020-01369-x

10. Dohlman JC, Lorch AC. Pivotal Trials in Ophthalmology: A Guide for Trainees. Springer Nature (2021). doi: 10.1007/978-3-030-63978-5

11. Medeiros FA, Jammal AA, Thompson AC. From machine to machine: An OCT-trained deep learning algorithm for objective quantification of glaucomatous damage in fundus photographs. Ophthalmology. (2019) 126:513-21. doi: 10.1016/j.ophtha.2018.12.033

12. Thompson AC, Jammal AA, Medeiros FA. A review of deep learning for screening, diagnosis, and detection of glaucoma progression. Trans Vis Sci Tech. (2020) 9:42. doi: 10.1167/tvst.9.2.42

13. Musch DC, Lichter PR, Guire KE, Standardi CL. The Collaborative Initial Glaucoma Treatment Study: study design, methods, and baseline characteristics of enrolled patients. Ophthalmology. (1999) 106:653-62. doi: 10.1016/S0161-6420(99)90147-1

14. Gedde SJ, Schiffman JC, Feuer WJ, Parrish RK, Heuer DK, Brandt JD. The tube versus trabeculectomy study: design and baseline characteristics of study patients. Am J Ophthalmol. (2005) 140:275-87. doi: 10.1016/j.ajo.2005.03.031

15. Christakis PG, Tsai JC, Zurakowski D, Kalenak JW, Cantor LB, Ahmed IIK. The ahmed versus baerveldt study: design, baseline patient characteristics, and intraoperative complications. Ophthalmology. (2011) 118:2172-9. doi: 10.1016/j.ophtha.2011.05.003

16. Gedde SJ, Chen PP, Heuer DK, Singh K, Wright MM, Feuer WJ, et al. Methodology of a multicenter randomized clinical trial comparing tube shunt surgery trabeculectomy with mitomycin C. Ophthalmology. (2018) 125:774-81. doi: 10.1016/j.ophtha.2017.1 0.037

17. Gazzard G, Konstantakopoulou E, Garway-Heath D, Barton K, Wormald R, Morris S, et al. Laser in Glaucoma and Ocular Hypertension (LiGHT) trial. A multicentre, randomised controlled trial: design and methodology. Br J Ophthalmol. (2018) 102:593-8. doi: 10.1136/bjophthalmol-2017-31 0877
18. King AJ, Fernie G, Azuara-Blanco A, Burr JM, Garway-Heath T, Sparrow JM, et al. Treatment of Advanced Glaucoma Study: a multicentre randomised controlled trial comparing primary medical treatment with primary trabeculectomy for people with newly diagnosed advanced glaucoma-study protocol. Br J Ophthalmol. (2018) 102:922-8. doi: 10.1136/bjophthalmol-2017-310902

19. Comparison of glaucomatous progression between untreated patients with normal-tension glaucoma and patients with therapeutically reduced intraocular pressures. Collaborative Normal-Tension Glaucoma Study Group. Am J Ophthalmol. (1998) 126:487-97. doi: 10.1016/S0002-9394(98)00223-2

20. Gordon MO, Kass MA. The Ocular Hypertension Treatment Study: design and baseline description of the participants. Arch Ophthalmol. (1999) 117:573-83. doi: 10.1001/archopht.117.5.573

21. Leske MC, Heijl A, Hyman L, Bengtsson B. Early Manifest Glaucoma Trial: design and baseline data. Ophthalmology. (1999) 106:2144-53. doi: 10.1016/S0161-6420(99)90497-9

22. Miglior S, Zeyen T, Pfeiffer N, Cunha-Vaz J, Torri V, Adamsons I. The European glaucoma prevention study design and baseline description of the participants. Ophthalmology. (2002) 109:161221. doi: 10.1016/S0161-6420(02)01167-3

23. Krupin T, Liebmann JM, Greenfield DS, Rosenberg LF, Ritch R, Yang JW. The Low-pressure Glaucoma Treatment Study (LoGTS) study design and baseline characteristics of enrolled patients. Ophthalmology. (2005) 112:37685. doi: 10.1016/j.ophtha.2004.10.034

24. Garway-Heath DF, Lascaratos G, Bunce C, Crabb DP, Russell RA, Shah A, et al. The United Kingdom Glaucoma Treatment Study: a multicenter, randomized, placebo-controlled clinical trial: design and methodology. Ophthalmology. (2013) 120:68-76. doi: 10.1016/j.ophtha.2012.07.028

25. Advanced Glaucoma Intervention Study. 2. Visual Field Test Scoring and Reliability. (1994). Available online at: https://pubmed.ncbi.nlm.nih.gov/ 7741836/ (accessed November 1, 2021).

26. Morgan RK, Feuer WJ, Anderson DR. Statpac 2 glaucoma change probability. Arch Ophthalmol. (1991) 109:16902. doi: 10.1001/archopht.1991.01080120074029

27. Greaney MJ, Hoffman DC, Garway-Heath DF, Nakla M, Coleman AL, Caprioli J. Comparison of optic nerve imaging methods to distinguish normal eyes from those with glaucoma. Investigative Ophthalmol Visual Sci. (2002) 43:140-5.

Conflict of Interest: The authors declare that the research was conducted in the absence of any commercial or financial relationships that could be construed as a potential conflict of interest.

Publisher's Note: All claims expressed in this article are solely those of the authors and do not necessarily represent those of their affiliated organizations, or those of the publisher, the editors and the reviewers. Any product that may be evaluated in this article, or claim that may be made by its manufacturer, is not guaranteed or endorsed by the publisher.

Copyright (C) 2022 AlRyalat, Ertel, Seibold and Kahook. This is an open-access article distributed under the terms of the Creative Commons Attribution License (CC BY). The use, distribution or reproduction in other forums is permitted, provided the original author(s) and the copyright owner(s) are credited and that the original publication in this journal is cited, in accordance with accepted academic practice. No use, distribution or reproduction is permitted which does not comply with these terms. 